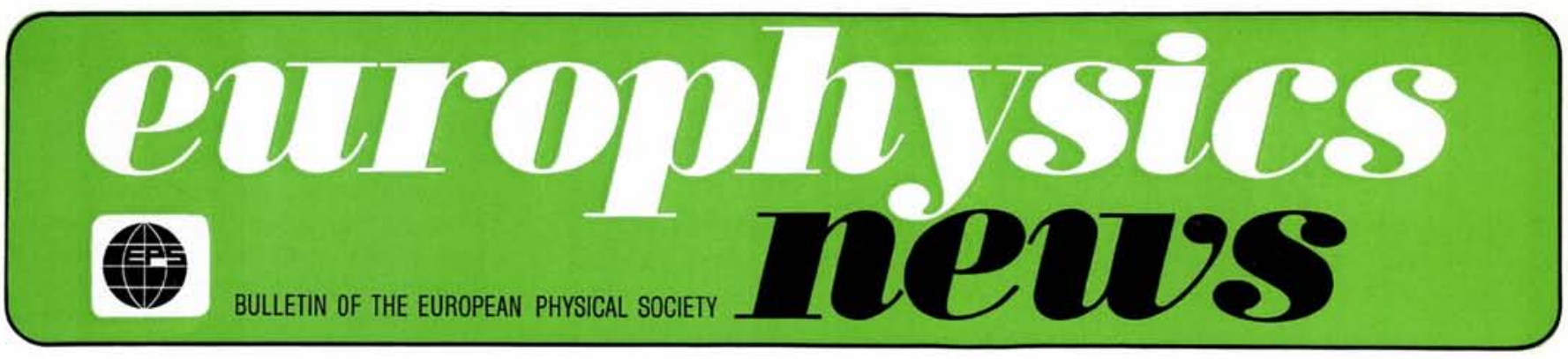

\title{
Applications of Liquid Crystals
}

\section{W. Helfrich, Berlin}

(Freie Universität)

Professor Helfrich is the 1976 winner of the Hewlett-Packard Europhysics Prize which is awarded annually for outstanding achievement in solid state physics. The award which is to be presented at the opening of the 19th. Congress Ampère in Heidelberg on 27 Sept., 1976 is for his contributions to the physics of liquid crystals, leading also to the discovery of the twisted nematic display and to a better understanding of certain biological phenomena.

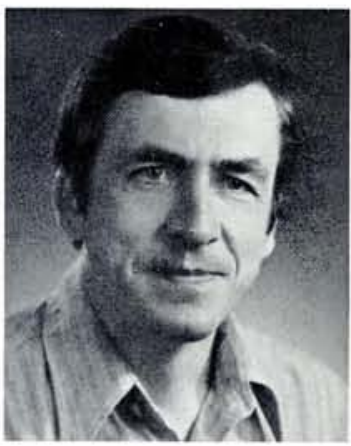

Most physicists today have probably heard of liquid crystals and have some vague idea of the meaning of this very descriptive term. Such general awareness did not seem to exist only ten years ago. For instance, when I was sent a preprint of some work on nematic liquids in 1967, neither I nor my colleagues in Munich understood what it was about. However, liquid crystals were discovered by the Austrian botanist F. Reinitzer') in 1888 and recognized for what they are by the German physicist $O$. Lehmann. The latter promoted the new state of matter not only devotedly but also aggressively which is, perhaps, one of the reasons why it took until 1922 for liquid crystals to become firmly established. In that year the French physicist G. Friedel published a classification of the most important types of these materials in terms of molecular arrangement. Interest in the physics of liquid crystals was kept alive and some important advances were made both before and after 1922 by a number of research scientists working mainly in France, Russia and Germany.

The modern era began with industrial research in the United States. In the early sixties, J. L. Fergason, then at Westinghouse, became interested in cholesteric liquid crystals because of their colour changes with temperature and R. Williams at RCA Laboratories discovered (or rediscovered) the so-called dynamic scattering of nematic liquid crystals in electric fields. The motive in both cases was to build thin optical displays. The work done and the reviews written by chemists strongly helped the physical studies until in the late sixties a growing number of physicists entered the field, a development culminating in, and highlighted by, P. G. de Gennes' recent book $^{2}$ ). Since then, electrooptic displays employing liquid crystals have become commonplace in electronic watches and pocket calculators, competing with light emitting diodes and other devices.

\section{Structure and Properties}

Liquid crystals are made up of elongated organic molecules. A famous example is the compound shown in Fig. 1 and simply called MBBA. The rigid core, often consisting of two benzene rings connected by some stiff bridge, and flexible end groups of variable length are typical. MBBA was the first one-component material, discovered in 1969, which is nematic at ambient rather than elevated temperatures.

As their name suggests, liquid crystals are at the same time anisotropic, like crystals, and fluid, like liquids; they also exist at temperatures between the solid and the liquid state.

Fig. 1. The molecular structure of 4-methoxy-4'- $n$-butylbenzylidene-aniline (MBBA), which forms a nematic liquid crystal between 21 and $48{ }^{\circ} \mathrm{C}$.

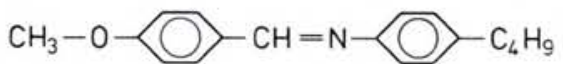

The basic structure is a more or less parallel arrangement of the molecules. If only the orientation of the long molecular axes is restricted but not translation, one has the nematic phase $^{3}$ ). It can be sheared like a fluid in all directions although with anisotropic viscosity. In a nematic phase containing chiral (i.e. optically active) molecules, the axis of preferential alignment usually rotates along and around another axis perpendicular to it, the pitch of the screw being never smaller than about $1000 \AA$. Such phases are denoted by a special name, cholesteric, because in their optical and mechanical properties they differ greatly from the nematic. For instance non-disruptive shear flow can take place only in directions perpendicular to the screw axis. Partial fluidity is also characteristic of smectic liquid crystals, where the molecules, apart from being parallel, are arranged in

\section{Contents}

Applications of Liquid Crystals

Heavy Nuclei and Super-heavy Elements

Letter to the Editor.

EGAS Conference Report .

News from the Divisions

8

Reduced Rates for I.O.M.s .

Europhysics News is published monthly by the European Physical Society. (c) 1976. Reproduction rights reserved. 


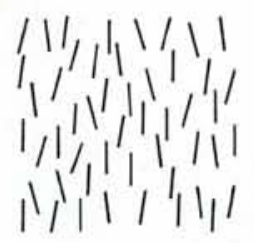

nematic

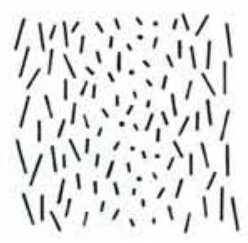

cholesteric

Fig. 2. Arrangement of molecules in the three basic liquid-crystalline phases. The arrow indicates the screw axis, also called helical axis, of the cholesteric structure.

usually monomolecular layers. Sketches of the three phases are shown in Fig. 2. Today several smectic phases are known to exist. In the least ordered state (called smectic A) the molecules are oriented normal to the layers which may be regarded as two-dimensional fluids. In others, the molecules are collectively tilted, form two-dimensional crystals that are free to slide along each other, etc. One compound may display a number of iıquid-crystalline states depending on temperature. The phase transitions involving liquid crystallinity are generally associated with strong pretransitional effects and some of them are of second order or near it.

From the point of view of continuum mechanics, liquid crystals are unique because of their easy deformability. The characteristic deformations, and the only ones in nematics and cholesterics, are orientational instead of translational. Three kinds can be distinguished: splay, twist, and bend of the orientation lines, as sketched in Fig. 3. Taking into account that each basic deformation has two components in three dimensions, one derives for the elastic energy density $g$ the expression

$$
g=K_{11}(\operatorname{div} \mathbf{n})^{2} / 2+
$$

$K_{22}\left(\mathbf{n} \text {. rot } \mathbf{n}+t_{0}\right)^{2} / 2+K_{33}(\mathbf{n} \Lambda \operatorname{rot} \mathbf{n})^{2} / 2$

where the unit vector $\mathbf{n}$ denotes the locally variable unique axis and the spontaneous twist $t_{0}$ is nonzero in cholesterics. (In general, liquid crystals are nonpolar. This is reflected in the formula for $g$ which is independent of the sign of $\mathbf{n}$.)

The elastic moduli $K_{11}, K_{22}$ and $K_{33}$ are of the order of $10^{-6}$ dyn. Accordingly, a uniform twist such that the alignment rotates by $90^{\circ}$ between the two bounding plates requires, for a $10 \mu \mathrm{m}$ thick layer, only an energy of about $10^{-3} \mathrm{erg} \mathrm{cm}^{-2}$. There is nothing mysterious about the smallness of the $K$ 's. Liquid crystalline deformations are expressed by the derivation of an angle with respect to a spatial coordinate, while compression and dilation are measured by the relative

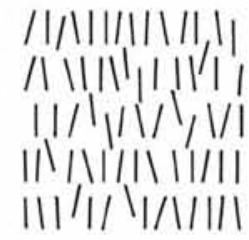

\section{smectic}

change of length. The latter is the same for macroscopic and molecular distances, while in the former case, tiny differences in the preferential alignment of neighbouring molecules add up to large angles at macroscopic distances.

\section{Technical Applications}

Somewhat related to the easy orientational deformability is the very sensitive response of the cholesteric pitch to changes in temperature and composition. The periodic structure, linked with the cholesteric twist gives rise to a kind of Bragg reflection. Consequently if the pitch is of the order of optical wavelengths, the colour of reflected light may be used as an indicator for these influences. In extreme cases, the colour goes from red to blue as the temperature is increased by only $1^{\circ} \mathrm{C}$. The dependence of pitch on temperature is at the root of liquid crystal thermometers and thermal mapping. Thermo-optic effects are used in medicine, material testing, and simple toys, such as plates that locally change colour at the touch of a finger.

Here I would like to say a little more about electro-optic displays, a broader subject both in a basic and an applied sense and one in which I was personally involved ${ }^{4}$ ). Nematics and cholesterics are used for this purpose and quite a number of different effects can be distinguished. Common to all is an electrical change of the orientation pattern or " texture " of the liquid crystal, affecting its appearance because of the inherent optical anisotropy of the material. The voltage is generally applied across a thin (ca. $10 \mu \mathrm{m})$ film of liquid crystal which is held between two glass plates provided with a conductive and transparent coat. Optical signals may be seen either in transmitted light or in light reflected at the back of the display cell. Usually one or two polarizers are needed to obtain a visible effect.

The simplest examples are probably the following two dielectric deformations. One first prepares what is called a single crystal by treating the bounding plates in such a way that they impose a uniform alignment, parallel or perpendicular to them, on the whole sample. This may be done by rubbing, etching, or vapour deposition of other materials on the coated surfaces. If the dielectric anisotropy of the liquid crystal has the right sign, an applied field will tend to turn the nematic axis into the other direction. The realignment occurs in the bulk of the sample while the orientation at the walls is essentially fixed, thus causing a restoring force which is very important for turn-off. The deformation sets in gradually above a threshold voltage of a few volts and is independent of film thickness. Physicists sometimes like to think of it as a field-induced second-order phase transition, the amplitude of the initially sinusoidal deformation being a possible order parameter. Such a picture permits a quick derivation of exact formulae for the threshold voltages, whose order may also be obtained by a crude comparison of elastic and dielectric energies. The difference of the two dielectric constants $\varepsilon_{\|}$and $\varepsilon_{+}$parallel and perpendicular

Fig. 3. The three liquid-crystalline deformations, showing splay, twist and bend of the orientation lines.
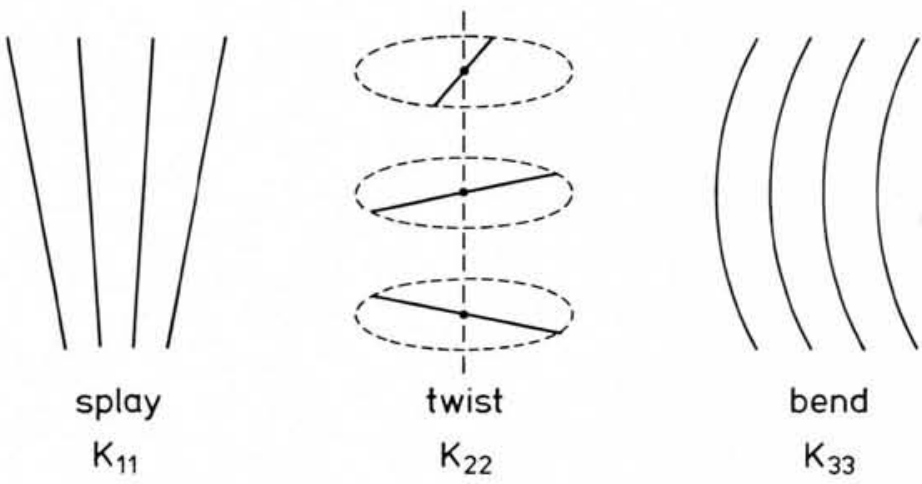
to the unique axis should be large to make the threshold voltage small. Materials with $\varepsilon_{\|}-\varepsilon_{\perp} \geqslant 15$ have, in fact, been synthesized.

For light propagating perpendicularly to the film, the two deformations just described convert a birefringent medium into an apparently isotropic one and vice versa. This can be exploited for electro-optic effects. However, a slightly more complicated arrangement is used in most of today's liquid crystal displays. The fixed wall alignment is parallel but differs by (almost) $90^{\circ}$ on the two plates so that the nematic sample is somewhat twisted and resembles a cholesteric. The electric vector of linearly polarized light travelling normal to the film rotates with the nematic axis, much like microwaves rotate with a waveguide. The rotation can be reduced to zero, often with voltages of the order of $1 \mathrm{~V}$, by an electric field which turns the nematic axis into its direction. Consequently, such a cell will go from dark to transparent when packed between parallel polarizers and from transparent to dark when the directions of polarization are orthogonal. In this context one often speaks of twist cells or the twisted-nematic effect.

There are other electro-optic effects which may be serious contenders and more challenging from a scientific point of view. For instance, a cholesteric-to-nematic "phase transition" can be brought about by dielectric realignment. Since the cholesteric pitch should be much smaller than the film thickness to preclude an influence of wall alignment, the phase transition requires comparatively large voltages. The enhancement factor as compared to the threshold of deformations in nematics equals roughly the square of the ratio of sample thickness to pitch. On the other hand, strongly dichroic cholesterics, as produced by the addition of suitable dye molecules aligning with the host, allow in principle electro-optic displays that do not require any polarizers.

Another class of liquid-crystalline deformations results from electro-hydrodynamic rather than dielectric instabilities and deformations. A pre-requisite is an anisotropic conductivity. Fairly small conductivities due to impurities or small concentrations of an organic salt are sufficient. An electric field will then create space charges in a distorted liquid crystal. As an example, we may consider a singlecrystalline nematic film due to a fixed wall alignment parallel to the bounding plates. Let us then imagine the orientation lines in the bulk to be deformed into periodic waves, the wave vector being parallel to the original alignment. The induced space charges of alternating sign will interact with the applied field and result in hydrodynamic flow, consisting of a sequence of vortices and involving shear. Shear flow in liquid crystals can exert a torque density competing with elastic and dielectric torques. Given the proper circumstances, the vortical flow associated with a thermal fluctuation of the orientation pattern will cause the fluctuation to grow into a stationary deformation rather than relax.

Periodic deformations of the kind envisaged are well known as Williams domains, they also have thresholds in the range of a few volts and a polarizer is helpful for visibility. At higher voltages, the liquid crystal becomes turbulent and scatters white light as does the powder of a transparent crystal. This is the so-called dynamic scattering mode whose turbulence is still very poorly understood. Further electro-optic effects are based on the electrohydrodynamic deformation of cholesterics. More recently, a large variety of hydrodynamic instabilities caused by heat flow and simple shearing have been observed in nematics and cholesterics.

\section{Connexion to Biophysics}

There seems to be a slowly but constantly growing interest in biophysics among physicists, perhaps because substantial advances in traditional areas have become more and more difficult. The question may arise if liquid crystals are a suitable starting point for such endeavours. I think they are in some respects. Lyotropic liquid crystals are noteworthy in this context and one of the reasons is that by definition, lyotropic phases are mixed systems which are liquid-crystalline in a restricted range of composition, as opposed to thermotropic liquid crystals which for their existence require only the right temperature.

Prominent examples are soap-water and lecithin-water systems: both have a lyotropic smectic phase where lipid bilayers alternate with thin layers of water. The alkyl chains make up the core of the bilayers while the polar heads of the molecules assemble at the two interfaces with water. In a large excess of water, the stacked bilayers of lecithins can be made to separate from each other and to form vesicles surrounded by one or more closed bilayers. Lecithin bilayers and their unilamellar vesicles are, in fact, frequently used as model systems for biological membranes and cells. However, certain measurements, such as NMR and ESR, cannot be done on a single lamella for reasons of sensitivity. A way to overcome these difficulties is to study the lyotropic smectic phase.

Lecithin bilayers can be two-dimensional solids or fluids, depending on temperature. This is most easily seen by the study of large enough vesicles under a phase contrast microscope ${ }^{5}$ ). The fluid phase possesses a curvature elasticity which is closely related to the splay elasticity of liquid crystals. The elastic energy $w$ per unit area may be written as

$$
w=*\left(c_{1}+c_{2}-c_{0}\right)^{2} / 2
$$

where $x \approx 10^{-12}$ erg is an elastic modulus, $c_{1}$ and $c_{2}$ the two principal curvatures, and $c_{0}$ is a spontaneous curvature that may arise if the two sides of the bilayer are different or facing different media. Elaborate numerical calculations are needed to calculate from this simple formula the possible shapes of vesicles surrounded by a thin fluid membrane. The only parameters determining the shape are membrane area (thought to be constant), enclosed volume, and spontaneous curvature. Theory and experiment seem to match perfectly in the case of lecithin vesicles. Some shapes of red blood cells, in particular the well-known biconcave disk normally observed in human blood, can also be explained in terms of curvature elasticity $^{6}$ ), but other factors, such as shear elasticity, may come into play in a true biological membrane.

\section{References and Footnotes}

1. For the history of liquid crystals see KELKER, H., Mol. Cryst. and Liq. Cryst. 21, (1973) 1.

2. de GENNES, P.G., The Physics of Liquid Crystals (Clarendon Press, Oxford), 1974. A recent review was written by STEPHEN, M.J. and STRALEY, J.P., Rev. Mod. Phys. 45 (1974) 617 ; for a short guide to technical and physicochemical applications, see: MEIER, G., SACKMANN, E. and GRABMAIER, J.G., Applications of Liquid Crystals (Springer Verlag, Berlin), 1975; for more physics see a forthcoming Supplement to Solid State Physics.

3. The designations derive from:

(1) to $v \eta \mu \alpha=$ thread, because nematics often display lines of singular alignment looking like threads;

(2) cholesterol, because some of its derivatives were the first known cholesterics;

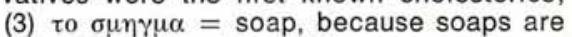
common lyotropic smectics.

4. For a brief review of electric alignment, see: HELFRICH, W., Liq. Cryst. and Mol. Cryst. 21 (1973), 187.

5. HARBICH, W., SERVUSS, R.M. and HELFRICH, W., Phys. Letters 57A (1976), 294. 6. DEULING. H.J. and HELFRICH, W., Biophys. J. in the press. 